\title{
Comparative effects of commonly used Artemisinin- based combination therapies (ACTs) on reproductive parameters in male wistar rats
}

\begin{abstract}
Malaria is an infectious disease of the blood caused by parasites called plasmodium, which infect red blood cells and are spread by mosquitoes. It is an endemic disease in Nigeria and many other African countries. Its prevalence is as follows: it affects 3.3 billion people in 106 countries worldwide. About 30 countries in sub-Saharan Africa account for 90 percent of global malaria deaths. It is the second leading cause of death in from infectious diseases after HIV/AIDS. The drugs recommended by the World Health Organisation (WHO) for the treatment and prevention of Malaria are the Artemisinin-based Combination Therapies (ACTs). They have been proven to be potent, with long lasting effects to effectively clear the plasmodium parasites from the blood system. ACTs are known to be well tolerated by the body in many countries, hence the recommendation by the WHO. The current research was to determine the comparative effects of short-term and prolonged use of two ACTs; (Artemether/ Lumefantrine and Artesunate/Amodiaquine) on the reproductive parameters in male wistar rats. The research determined the effects of the drugs on the various male reproductive hormones on administration of the drugs for 3 days (short-term) and for 6 days (prolonged use). Results showed that the ACTs did not have any significant/ deteriorative effect on the male reproductive parameters in the rats when used for short and long prolonged. It therefore implies that short-term and prolonged use of the two ACTs does not have deteriorative effect on male reproductive parameters. It however does not give a license for the drugs to be indiscriminately used and abused. It is only a guide to medical practitioners to consider repeating treatment on patients in cases where the parasites are not completely cleared from the system after first episode of treatment.
\end{abstract}

Keywords: plasmodium, acts, reproductive parameters, hormones, artemisinin, malaria

\author{
Volume 5 Issue 2 - 2018
}

\author{
Seriki A Samuel, Dare Ayobami, Allahyafi E \\ Jane \\ Department of Human Physiology, Bingham University, Nigeria
}

Correspondence: Seriki A Samuel, Department of Human Physiology, College of Medicine, Bingham University, Karu, Nigeria, Tel +234803604 I I 2 I, Email seriki.adinoyi@gmail.com

Received: March 24, 2018 | Published: April 16, 2018
Abbreviations: ACTs, artemisinin-based combination therapies; WHO, world health organisation; FSH, follicle-stimulating hormone; LH, luteinizing hormone; CT, combination therapy; PMS, post-marketing surveillance; ABPs, androgen-binding proteins; $\mathrm{GnRH}$, gonadotropin-releasing hormone; ICSH, interstitial cellstimulating hormone; MAO, monoamine oxidase

\section{Introduction}

Malaria is a major public health problem in Nigeria and other African countries. It is an infectious disease of the blood caused by Plasmodium parasites which infect red blood cells and are spread by mosquitoes. There are two main types of malaria; Falciparum malaria, which can be lethal, is the more serious form. Vivax malaria is better known for its ability to relapse several months to years after an infected person has left a malarious area, due to residual parasites in the liver. The World Health Organization has estimated that each year, there are about 250 million cases of malaria leading to approximately one million deaths, mostly in children under five years of age. The organism that causes the most dangerous form of malaria is a microscopic parasite called Plasmodium falciparum. This parasite is transmitted by mosquito species belonging to the Anopheles genus and only by females of those species. ${ }^{1}$
The most common symptoms of malaria include influenza-like illness that rapidly develops hectic fevers. It is characterised by cycles of chills, fever, pain and sweating. Some patients develop nausea, vomiting, coughs and diarrhoea. Cycles of chills, fever and sweating that repeat every one or two days, are typical. The basics of malaria prevention are well known: avoid mosquito bites; and take antimalarial medications for prevention when exposed (also known as known as chemoprophylaxis). The use of mosquito repellants and insecticides has also helped in preventing malaria. Clearing mosquito breeding sites such as bushes and stagnant waters from environment, as well as use of insecticide-treated nets have also been identified to help prevent infection.

\section{Artemisinin-based combination therapies (ACTs)}

Artemisinin and its derivatives are powerful medicines known for their ability to swiftly reduce the number of Plasmodium parasites in the blood of patients with malaria. Artemisinin-based combination therapies (ACTs) are the best anti-malarial drugs currently available in the market. Artemisinin enhances efficacy and has the potential of lowering the rate at which resistance emerges and spreads. Under low transmission intensity, ACTs have an additional public health benefit of reducing the overall malaria transmission. ACTs are recommended 
by $\mathrm{WHO}$ as the first-line treatment for uncomplicated $P$. falciparum malaria. Expanding access to ACTs in malaria-endemic countries has been integral to the remarkable recent success in reducing the global malaria burden. The number of ACT treatment courses procured from manufacturers increased globally from 187 million in 2010 to 311 million in $2015 .^{2}$

ACTs combine artemisinin derivatives with a partner drug. The role of the artemisinin compound is to reduce the main parasite load during the first 3 days of treatment, while the role of the partner drug is to eliminate the remaining parasites. In patients who are infected with artemisinin-resistant strains of malaria, the artemisinin compound is less effective in clearing all parasites within this 3-day period. However, patients are still cured as part of a longer treatment regimen, provided that they are treated with an ACT containing a partner drug that is effective in that geographical area. WHO currently recommends at least five different ACTs. They are ArtemetherLumefantrin, Artesunate-Amodiaquine, Artesunate-Mefloquine, Artesunate-sulfadoxine/ pyrimethamine, Artesunate-piperaquine and Artesunate-chlorproguanil/dapsone. ${ }^{2}$

Despite being recommended by WHO since 2001, overall deployment of ACT has been slow. Limiting factors are high cost, limited knowledge and public awareness on the concept of combination therapy (CT) and ACT in particular, limited knowledge on safety of ACTs in pregnancy, operational issue such as inappropriate drug use, lack of suitable drug formulations, lack of post-marketing surveillance (PMS) systems, and the imbalance between demand and supply. Through concerted efforts of multilateral organizations, the local scientific community with involvement of policy-makers progress has been on several fonts leading to improved ACT uptake rates in the last 2 years. ${ }^{3}$

Of 43 countries that had adopted ACT by February 2005, 18 (42\%) adopted the policy in 2004. Preference to co-formulated Coartem has led to a surge in its demand with consequent shortage. Alternative ways for increased production of ACTs are urgently needed otherwise most policies will remain adopted on paper. ${ }^{3}$

\section{Reproductive parameters}

The male reproduction parameters are mainly measured in terms of blood level of testosterone, follicle-stimulating hormone (FSH), luteinizing hormone (LH) and sometimes the weight and volume of the testis. ${ }^{4}$

\section{Testosterone}

Testosterone is a steroid hormone that is the most potent naturally occurring androgen that regulates the development of the male reproductive system and male secondary sex characteristics. It is produced primarily by the testes/testicles in cells called the Leydig cells. Females also produce testosterone, but usually in smaller amounts. Chemical formula: $\mathrm{C}_{19} \mathrm{H}_{28} \mathrm{O}_{2} \cdot{ }^{5}$

Without adequate testosterone, men are infertile. This is because the process of spermatogenesis (development of mature sperm) requires testosterone. In men, testosterone is thought to regulate a number of functions in addition to sperm production. They include sex drive, bone mass fat distribution, muscle size and strength, red blood cell production. Therefore low levels of testosterone have been implicated in decreased desire for sex (libido), diminished erectile quality (particularly for night-time erections), changes in mood, reduced intellectual and cognitive function, fatigue, depression and anger, and decrease in muscle mass/strength. ${ }^{5}$

\section{Follicle-stimulating hormone (FSH)}

Follicle-stimulating hormone (FSH) is a gonadotropin, a glycoprotein polypeptide hormone. FSH is synthesized and secreted by the gonadotropic cells of the anterior pituitary gland, and regulates the development, growth, pubertal maturation, and reproductive processes of the body. FSH and luteinizing hormone (LH) work together in the reproductive system.

FSH regulates the development, growth, pubertal maturation and reproductive processes of the human body. In both males and females, FSH stimulates the maturation of germ cells. In males, FSH induces Sertoli cells to secrete androgen-binding proteins (ABPs), regulated by inhibin's negative feedback mechanism on the anterior pituitary. Specifically, activation of Sertoli cells by FSH sustains spermatogenesis and stimulates inhibin B secretion. ${ }^{6}$

GnRH has been shown to play an important role in the secretion of FSH, with hypothalamic-pituitary disconnection leading to a cessation of FSH. GnRH administration leads to a return of FSH secretion. FSH is subject to oestrogen feed-back from the gonads via the hypothalamic pituitary gonadal axis. FSH stimulates primary spermatocytes to undergo the first division of meiosis, to form secondary spermatocytes. It enhances the production of androgenbinding protein by the Sertoli cells of the testes by binding to FSH receptors on their basolateral membranes, ${ }^{6}$ and is critical for the initiation of spermatogenesis.

\section{Luteinizing hormone (LH)}

Luteinizing hormone (LH) is a hormone produced by gonadotropic cells in the anterior pituitary gland.. In males, where LH had also been called interstitial cell-stimulating hormone (ICSH), it stimulates Leydig cell production of testosterone. It acts synergistically with FSH. LH acts upon the Leydig cells of the testis and is regulated by gonadotropin-releasing hormone $(\mathrm{GnRH})$. The Leydig cells produce testosterone $(\mathrm{T})$ under the control of $\mathrm{LH}$, which regulates the expression of the enzyme 17 $\beta$-hydroxysteroid dehydrogenase that is used to convert androstenedione, the hormone produced by the testes, to testosterone, ${ }^{7}$ an androgen that exerts both endocrine activity and intratesticular activity on spermatogenesis. ${ }^{8}$

LH is released from the pituitary gland, and is controlled by pulses of gonadotropin-releasing hormone. When testosterone levels are low, $\mathrm{GnRH}$ is released by the hypothalamus, stimulating the pituitary gland to release LH. As the levels of testosterone increase, it will act on the hypothalamus and pituitary through a negative feedback loop and inhibit the release of GnRH and LH consequently. Androgens (T, DHT) inhibit monoamine oxidase (MAO) in pineal, leading to increased melatonin and reduced LH and FSH by melatonin-induced increase of $\mathrm{GnIH}$ synthesis and secretion. T can also be aromatized into estradiol (E2) to inhibit LH. E2 decreases pulse amplitude and responsiveness to GnRH from the hypothalamus onto the pituitary. ${ }^{8}$

WHO recommends artemisinin-based combination therapies (ACTs) for the treatment of uncomplicated malaria caused by the $P$. falciparum parasite. By combining two active ingredients with different mechanisms of action, ACTs are the most effective antimalarial medicines available today, but they certainly leave some adverse effect on the patients. 
These ACTs and some other drugs used in the treatment of malaria infections have been reported to have some impacts on some haematological parameters of the patients due to their toxic effects, especially on the red blood cells (RBC). However, not so much have been done on the possible effects of some of these ACTs on the reproductive parameters of the patients. The present study does not only consider the effects of these ACTs on the reproductive parameters of the male wistar rats but also compares the effects of two ACTs with each other on the male reproductive parameters. The current study investigates the comparative effects of the ACTs on the reproductive parameters in male wistar rats.

The study also compares the effect of short-term use and prolonged use of the drugs by comparing the level of these hormones in the groups treated with the drugs for three (3) days and those treated for six (6) days to determine if prolonged treatment will impact any different effect on the hormones. The study compares the weight and volume of the testes and other vital organs of the body of the treated groups to the control.

\section{Clinical features of malaria}

The signs and symptoms of malaria typically begin 8 to 25 days following infection. However, symptoms may occur later in those who have taken antimalarial medications as prevention. ${ }^{9}$ Initial manifestations of the disease common to all malaria species are similar to flu-like symptoms, and can resemble other conditions such as sepsis, gastroenteritis, and viral diseases. ${ }^{7}$ The presentation may include headache, fever, shivering, joint pain, vomiting, hemolytic anemia, jaundice, hemoglobin in the urine, retinal damage, and convulsions. ${ }^{10}$

The classic symptom of malaria is paroxysm- a cyclical occurrence of sudden coldness followed by shivering and then fever and sweating, occurring every two days (tertian fever) in $P$. vivax and $P$. ovale infections, and every three days (quartan fever) for $P$. malariae. $P$. falciparum infection can cause recurrent fever every $36-48$ hours, or a less pronounced and almost continuous fever.

Severe malaria is usually caused by $P$. falciparum (often referred to as falciparum malaria). Symptoms of falciparum malaria arise 9 to 30 days after infection. Individuals with cerebral malaria frequently exhibit neurological symptoms, including abnormal posturing, nystagmus, conjugate gaze palsy (failure of the eyes to turn together in the same direction), opisthotonus, seizures, or coma. ${ }^{11}$ Individuals with cerebral malaria frequently exhibit neurological symptoms, including abnormal posturing, nystagmus, conjugate gaze palsy (failure of the eyes to turn together in the same direction), opisthotonus, seizures, or coma.

\section{Complications}

Malaria has several serious complications. Among these is the development of respiratory distress, which occurs in up to $25 \%$ of adults and $40 \%$ of children with severe P. falciparum malaria. Possible causes include respiratory compensation of metabolic acidosis, noncardiogenic pulmonary oedema, concomitant pneumonia, and severe anaemia. Although rare in young children with severe malaria, acute respiratory distress syndrome occurs in 5 to $25 \%$ of adults and up to $29 \%$ of pregnant women. ${ }^{10}$ Co-infection of HIV with malaria increases mortality. Renal failure is a feature of black water fever, where hemoglobin from lysed red blood cells leaks into the urine. ${ }^{11}$
Infection with $P$. falciparum may result in cerebral malaria, a form of severe malaria that involves encephalopathy. It is associated with retinal whitening, which may be a useful clinical sign in distinguishing malaria from other causes of fever. ${ }^{12}$ Enlarged spleen, enlarged liver or both of these, severe headache, low blood sugar, and hemoglobin in the urine with renal failure may occur. Complications may include spontaneous bleeding, coagulopathy, and shock. ${ }^{12}$ Malaria in pregnant women is an important cause of stillbirths, infant mortality, abortion and low birth weight, particularly in $P$. falciparum infection, but also with $P$. Vivax. ${ }^{13}$

\section{Materials and methods}

\section{Materials}

Well-ventilated animal cages, Wood fillings, Feeders and drinkers, Standard rat chow and distill water, Oral cannula, Rubber gloves, Weighing scale, Artesunate-amodiaquine (brand name; Anate), Artemether-lumefantrine (brand name; Lonart), Needles and syringes, Serum bottles, Centrifuge and Pasteur pipette, Fortress ELISA kit for testosterone, FSH and LH.

\section{Drug and chemicals}

Artemether + Lumefantrine (Lonart) and Artesunate + Amodiaquine (Anate) Bliss Gvs Pharma Ltd., India, and Adams Pharmaceutical Co. Ltd. (China) were purchased from New Health Pharmacy, Abuja. ELISA kit for testosterone, Luteinizing hormone and Follicle Stimulating Hormone (FSH) were obtained from Fortress manufacturer, UK. All other chemicals and reagents used were of analytical grade.

\section{Experimental animals}

A total of twenty five (25) male albino rats (90-140g) were used for the study. The animals are purchased from the Central Animal House of the College of Medicine, University of Ibadan. The rats were provided with feeds and water ad libitum all through the period of the study. They were housed in well-ventilated cages under a photoperiod controlled environment (12L:12D cycles) with temperature range of $25-27^{\circ} \mathrm{C}$ at the Animal house, College of Medicine, Bingham University, Karu. They were allowed to acclimatize for two weeks before administration of drugs. The drug was administered to the rats orally by the use of oral cannula. All the animals were cared for in accordance with the 'Guide for the Care and Use of Laboratory Animals' (1996).

\section{Experimental design}

The dose of Lonart (artemether/lumefantrine, 20/120mg/kg) and Anate (artesunate-amodiaquine $100 / 300 \mathrm{mg} / \mathrm{kg}$ ) used for this study was calculated from the manufacturer's recommended dose for a man weighing at least $35 \mathrm{~kg}$. Treatment was provided in all groups two times daily (every 12 hours) at therapeutic doses. The animals were randomly divided into five (5) groups of five animals each and treated as follows:

a. Group A served as control and was administered with distilled water.

b. Group B was administered therapeutic dose of ArtesunateAmodiaquine $(2.86 / 8.58 \mathrm{mg} / \mathrm{kg})$ for 3 Days. ${ }^{14}$

c. Group C was administered therapeutic dose of Artemether/ Lumefantrine $(1.14 / 6.86 \mathrm{mg} / \mathrm{kg})$ for 3 days. 
d. Group D was administered therapeutic dose of artesunateamodiaquine, $(2.86 / 8.58 \mathrm{mg} / \mathrm{kg})$ for 6 days.

e. Group E was administered therapeutic dose of artemeterlumefantrine $(1.14 / 6.86 \mathrm{mg} / \mathrm{kg})$ for 6 days.

f. Drug Preparation and Administration.

The drugs used in this study were grinded to a powdered form, mixed with distilled water and administered as an aqueous suspension. The drug suspension was continuously agitated during administration in order to deliver the drug homogeneously to the animals. The respective treatments were administered to all rats in all groups twice daily for a period of three and six days respectively using a $1.0 \mathrm{~mL}$ syringe by oral gavage. The choice of dose and route of administration was done as recommended for humans, but the six days duration of treatment was to expose the animals to treatment beyond the recommended duration, to mimic the extended duration of treatment associated with malaria relapse due to incomplete treatment or endemicity.

\section{Blood and organ collection}

The Animals were weighed on day 1,4 and 7. They were sacrificed 12 hours after the last administration (i.e. day 4 and 7) by cervical dislocation. Blood was collected by cardiac puncture into a plain serum bottle and later centrifuged at $3500 \mathrm{rev} / \mathrm{min}$ at room temperature for $15 \mathrm{~min}$ to obtain serum. The serum was kept frozen until it was used for hormonal analysis. The reproductive organs (testes, seminal vesicle, epididymis and prostate), liver and kidney were carefully harvested. They were immediately cleared of adhering tissues and weighed.

\section{Hormonal assay}

The serum samples obtained were analyzed to determine the concentration of testosterone, Luteinizing hormone and Follicle Stimulating Hormone. The analysis was done using stat-fax semiautomatic machine (STAT-FAX 3300, Awareness Technology Inc. 2001) and cobas e411 auto-analyzer (cobas e411/2006/Japan). The reagents used were Accubind ELISA reagents. Serum samples were assayed for levels of Testosterone, FSH and LH using Microwell Enzyme Linked Immuno Assay (ELISA) technique. The optical density was read at wavelength of between 492 and $550 \mathrm{~nm}$ using a spectrophotometer.

\section{Statistical analysis}

Data are presented as mean \pm standard error of mean (SEM) difference between groups was analyzed using student's t-test and one-way analysis of variance (ANOVA) where necessary. Values were considered statistically significant at $\mathrm{p}<0.05$.

\section{Results}

\section{Effect ACTs on reproductive hormones}

\section{Effect on testosterone}

Treatment of male rats with artesunate-amodiaquine (ats-amq) and artemether-lumefantrine (art-lum) for 3 and 6 days caused no statistically significant difference in the serum level of testosterone in the treatment group when compared with the control. Also, the 3 days and 6 days treatment shows no significant difference when compared with each other (Figure 1).

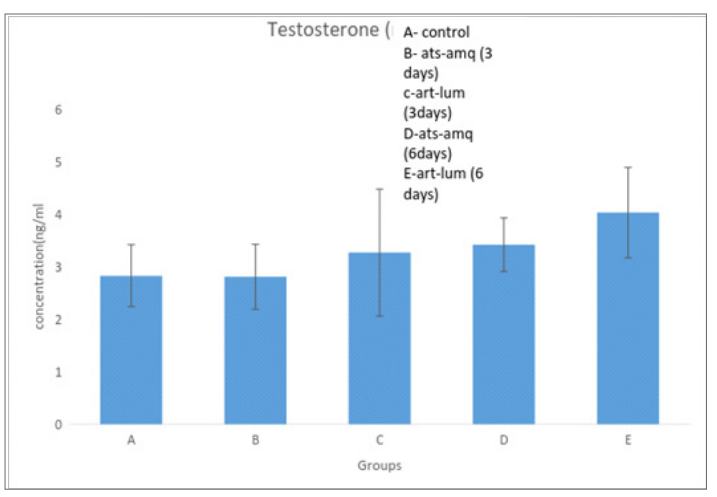

Figure I Effect of artesunate-amodiaquine (ats-amq) and artemetherlumefantrine (art-lum) on serum level of testosterone in male rats. $* \mathrm{P}<0.05$ when compare with control. $\mathrm{AP}<0.05$ when compared with day 3 .

\section{Effect on luteinizing hormones (LH)}

Treatment of male rats with artesunate-amodiaquine (ats-amq) and artemether-lumefantrine (art-lum) for 3 and 6 days caused no statistically significant difference in the serum level of luteinizing hormone in the treatment group when compared with the control. Also, the 3 days and 6 days treatment shows no significant difference when compared with each other (Figure 2).

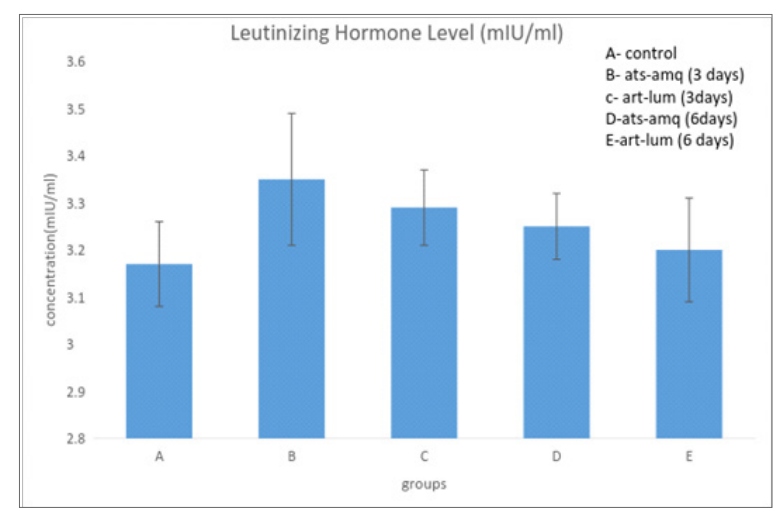

Figure 2 Effect of artesunate-amodiaquine (ats-amq) and artemetherlumefantrine (art-lum) on serum level of luteinizing hormone of male rats. $* \mathrm{P}<0.05$ when compare with control. $\mathrm{AP}<0.05$ when compared with day 3 .

\section{Effect on follicle stimulating hormone (FSH)}

Treatment of male rats with artesunate-amodiaquine (ats-amq) and artemether-lumefantrine (art-lum) for 3 and 6 days caused no statistically significant difference in the serum level of Follicle Stimulating Hormone (FSH) in the treatment group when compared with the control. Also, the 3 days and 6 days treatment shows no significant difference when compared with each other (Figure 3).

\section{Effects of ACTs on body weight}

Treatment of male rats with both artesunate-amodiaquine (atsamq) and artemether-lumefantrine (art-lum) for 3 and 6 days caused no statistically significant difference in the body weight of the 
treatment group when compared with the control. Also, the 3 days and 6 days treatment shows no significant difference when compared with each other (Figure 4).

\section{Effects of ACTs on organ weight}

\section{Effect on Weight of Testes}

Treatment of male rats with artesunate-amodiaquine (ats-amq) and artemether-lumefantrine (art-lum) for 3 and 6 days caused no statistically significant difference in the testicular weight of the control and treatment groups. Also, the 3 days and 6 days treatment shows no significant difference when compared with each other (Figure 5).

\section{Effect on weight of epididymis}

Treatment of male rats with artesunate-amodiaquine (ats-amq) and artemether-lumefantrine (art-lum) for 3 and 6 days caused no statistically significant difference in the epididymal weight of the control and treatment groups. Also, the 3 days and 6 days treatment

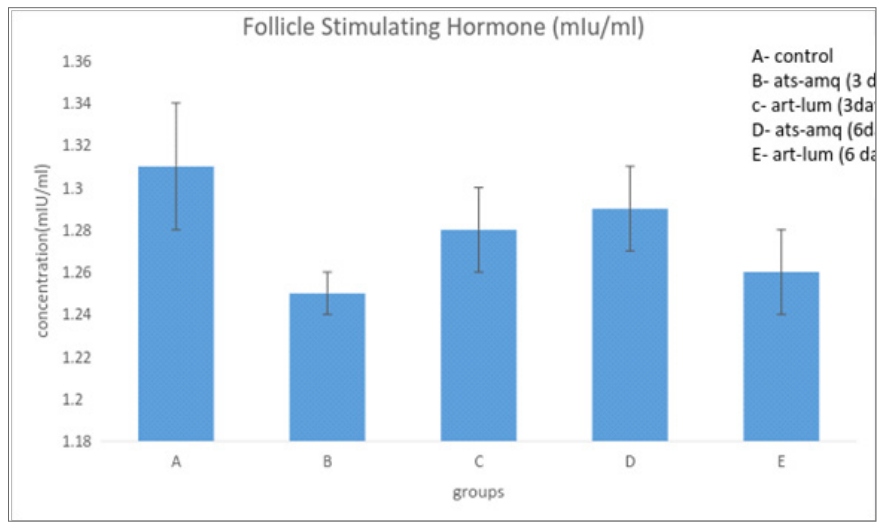

Figure 3 Effect of atesunate-amodiaquine (ats-amq) and artemetherlumefantrine (art-lum) on serum level of Follicle stimulating hormone (FSH) of male rats.

$* \mathrm{P}<0.05$ when compare with control. \#P<0.05 when compared with day 3 .

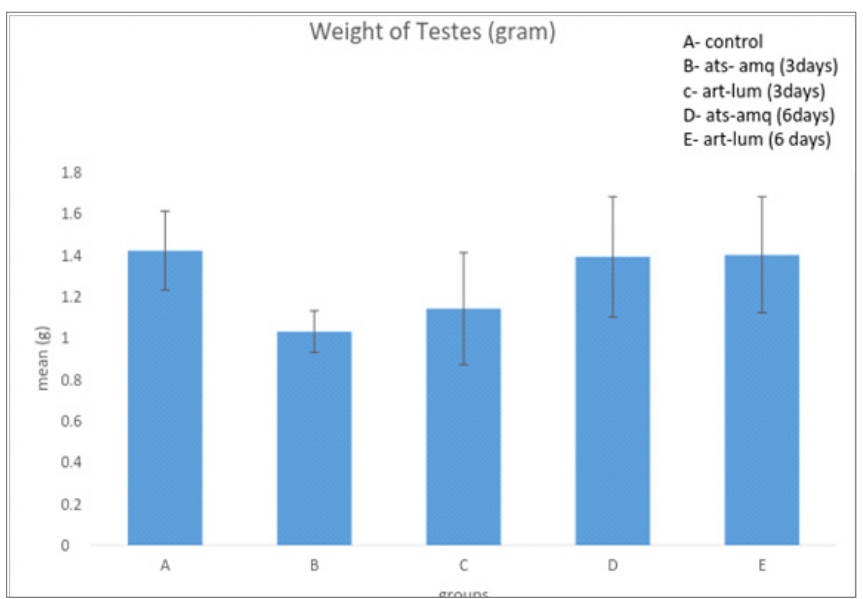

Figure 5 Effect of artesunate-amodiaquine (ats-amq) and artemetherlumefantrine (art-lum) on testicular weight of male rats.

$* \mathrm{P}<0.05$ when compare with control. $\# \mathrm{P}<0.05$ when compared with day 3 . showed no significant difference when compared with each other (Figure 6).

\section{Effect on weight of prostate}

Treatment of male rats with artesunate-amodiaquine (ats-amq) and artemether-lumefantrine (art-lum) for 3 and 6 days caused no statistically significant difference in the weight of prostate of the control and treatment groups. Also, the 3 days and 6 days treatment showed no significant difference when compared with each other (Figure 7).

\section{Effect on weight of seminal vesicle}

Treatment of male rats with artesunate-amodiaquine (ats-amq) and artemether-lumefantrine (art-lum) for 3 and 6 days causes no statistically significant difference in the weight of seminal vesicle of the control and treatment groups. Also, the 3 days and 6 days treatment shows no significant difference when compared with each other (Figure 8).

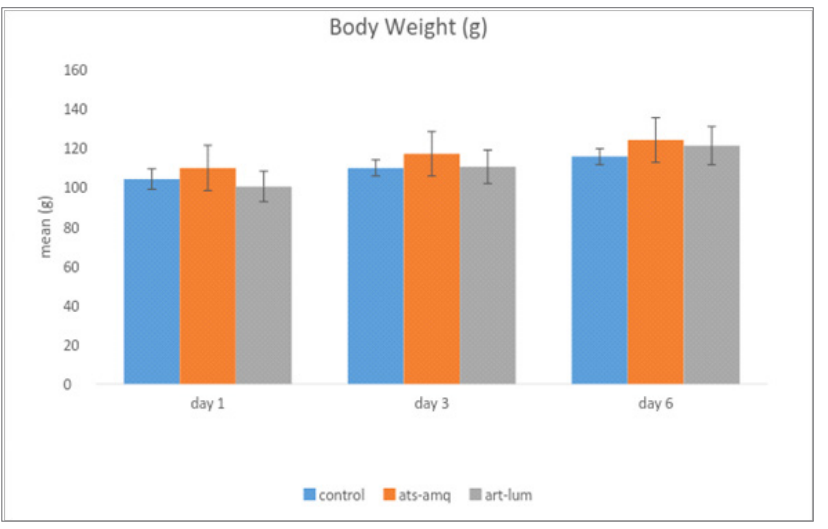

Figure 4 Effect of artesunate-amodiaquine (ats-amq) and artemetherlumefantrine (art-lum) on body weight of male rats.

$* \mathrm{P}<0.05$ when compare with control. \#P<0.05 when compared with day 3 .

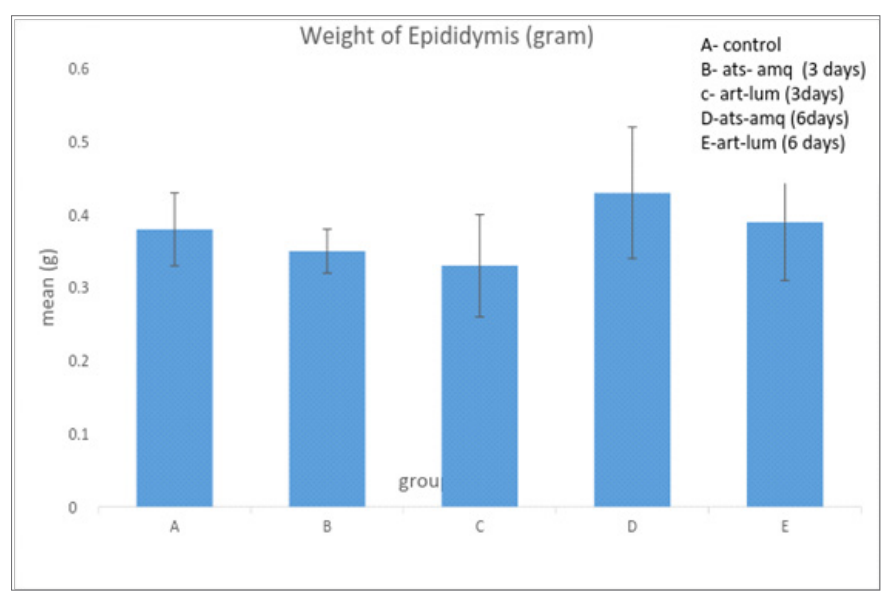

Figure 6 Effect of artesunate-amodiaquine (ats-amq) and artemetherlumefantrine (art-lum) on epididymal weight of male rats. $* \mathrm{P}<0.05$ when compare with control. $\# \mathrm{P}<0.05$ when compared with day 3 . 


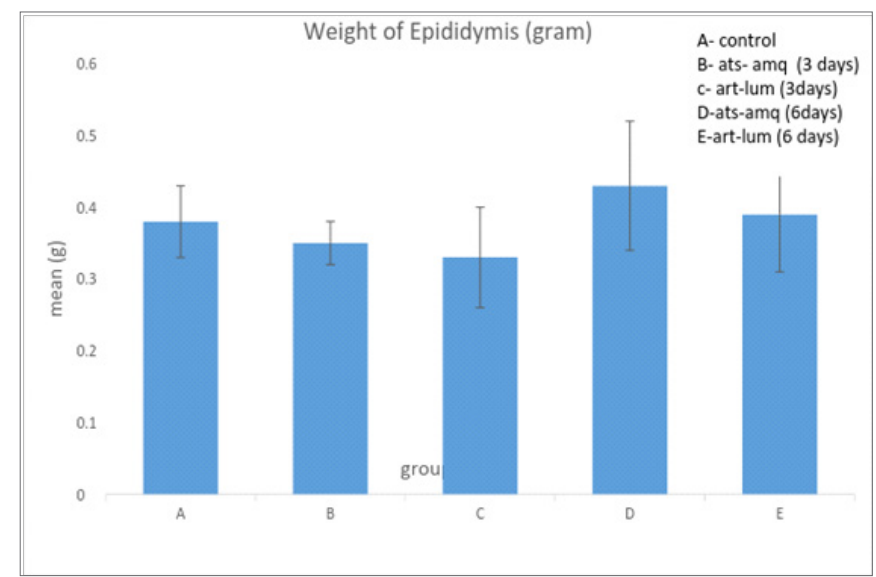

Figure 6 Effect of artesunate-amodiaquine (ats-amq) and artemetherlumefantrine (art-lum) on epididymal weight of male rats.

$* \mathrm{P}<0.05$ when compare with control. \#P<0.05 when compared with day 3 .

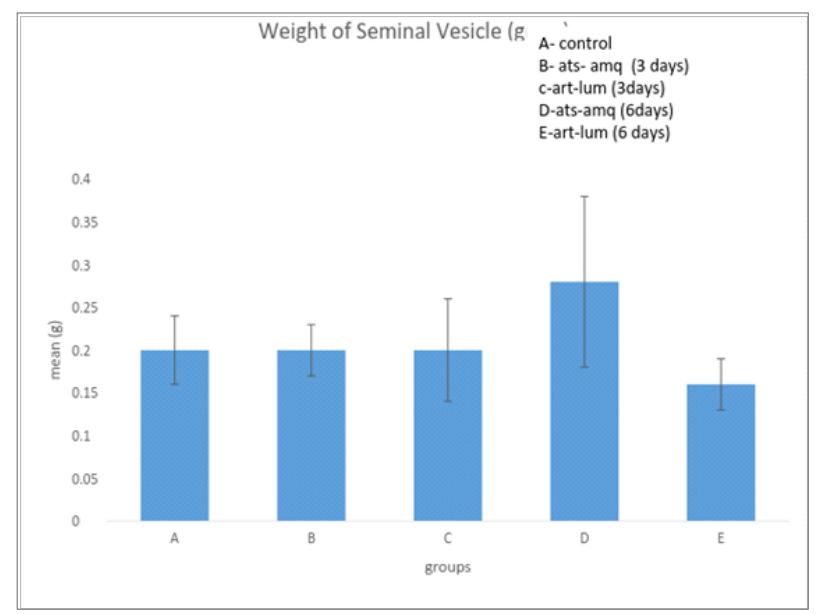

Figure 8 Effect of atesunate-amodiaquine (ats-amq) and atemetherlumefantrine (art-lum) on weight of seminal vesicle in male rats.

$* \mathrm{P}<0.05$ when compare with control. $\# \mathrm{P}<0.05$ when compared with day 3 .

\section{Discussion}

Many anti-malarial drugs and antibiotic agents have been reported to have anti-fertility actions. For instance the anti-steroidogenic and anti-fertility actions of quinine and chloroquine have been well documented. ${ }^{15,16}$ The effects of the anti-malarial (quinine), on peripheral level of testosterone, gonadotrophin and sperm motility have also been studied. With the increased efforts in the development of more potent anti-malarial agents as a result of the challenges posed by the resistant strains of the malaria parasite, the evaluation of these anti-malarial agents for possible anti-fertility actions in males becomes imperative since both malaria and infertility are worldwide phenomena and there is need to avoid the risk of infertility resulting from malaria chemotherapy.

In the current study, the comparative effects of two Artemisininbased Combination Therapies (ACTs); artemether-lumefantrine and artesunate-amodiaquine, on the reproductive parameters of the male wistar rats were investigated. The levels of testosterone, Follicle Stimulating Hormone (FSH) and Luteinizing Hormone (LH), which

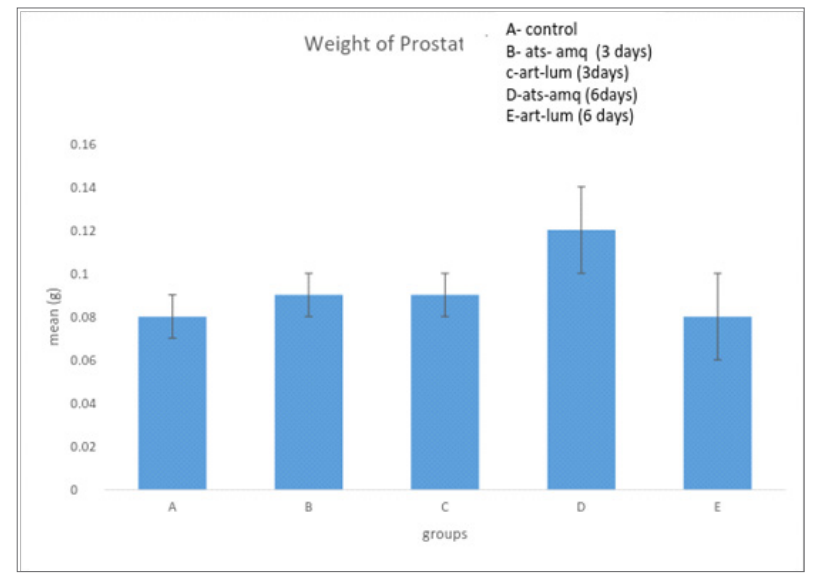

Figure 7 Effect of atesunate-amodiaquine (ats-amq) and atemetherlumefantrine (art-lum) on weight of the prostate in male rats.

$* \mathrm{P}<0.05$ when compare with control. \#P<0.05 when compared with day 3 .

are the major male reproductive hormones, and which combine to enhance spermatogenesis, ${ }^{17}$ were compared in the treated groups and with the control group.

Testosterone is particularly necessary to give growth to the Sertoli cells that in turn give support and nourishment to the germ cells and the produced spermatozoa. ${ }^{17}$ In the male, LH stimulates testosterone production from the interstitial cells of the testes (Leydig cells). FSH stimulates testicular growth and enhances the production of an androgen-binding protein by the Sertoli cells, which are a component of the testicular tubule necessary for sustaining the maturing sperm cell. This androgen-binding protein causes high local concentrations of testosterone near the sperm, an essential factor in the development of normal spermatogenesis. Sertoli cells, under the influence of androgens, also secrets inhibin, a polypeptide, which may help to locally regulate spermatogenesis. Hence, maturation of spermatozoa requires $\mathrm{FSH}$ and $\mathrm{LH}^{18}$

The results of the current study show that administration of artemether-lumefantrine and artesunate-amodiaquine for 3 days and for 6 days did not cause any significant effect on the level of testosterone, FSH and LH, and by implication will not cause any significant effect on the male reproductive parameters in rats, even when administration is prolonged beyond the normal 3-day administration by 3 additional days. Also, the results agreed with findings by Obianime and Aprioku that artesunate and amodiaquine separately decreased serum FSH and LH level significantly, but artesunate/amodiaquine combination caused only moderate effects which are not significant. This therefore indicates that artesunate will ameliorate the adverse effects of amodiaquine on LH and FSH when used together. ${ }^{19}$

Most importantly (for this study), there was no significant difference between the levels of the hormones (testosterone, FSH, and LH) when the groups administered with the two types of ACTs (artemetherlumefantrine and artesunate-amodiaquine) were compared with each other. This infers that the two ACTs under consideration are not in variance in their effects on male reproductive hormones, and will not cause significant adverse effect on the male reproductive hormones.

The current study also revealed no significant reduction in the organ weight (testes, epididymis, prostate, and seminal vesicle) of the rats treated with the ACTs when compared with control, indicatjng 
that the drugs possibly have no significant adverse effects on sperm production, testicular function or male fertility when administered at clinical dosage for 3 and 6 days. This also supports the results observed with the effect on the level of hormones.

\section{Conclusion}

Artemether-Lumefantrin and Artesunate-Amodiaquine, when administered at doses of $1.14 / 6.86 \mathrm{mg} / \mathrm{kg}$ and $2.86 / 8.58 \mathrm{mg} / \mathrm{kg}$ respectively, for 3 and 6 days did not cause any significant adverse effect on male reproductive parameters (testosterone, FSH and LH levels). This implies that the ACTs, given at clinical dosage for 3 and 6 days will not affect reproductive parameters adversely. The drugs can therefore be used at clinical dosage to treat malaria for 3 and 6 days without fear of any significant adverse effects on male reproductive parameters.

This lends credence to WHO's choice of ACTs as the first line drug for the treatment of malaria, which minimal side effects. Also, there was no significant difference between the effects of ArtemetherLumefantrin when compared with Artesunate-Amodiaquine on reproductive parameters. The two ACTs showed similar effects on reproductive parameters. There is no variance in the effects of the two ACTs on male reproductive parameters.

\section{Recommendations}

Similar studies should be conducted in human and in the females to observe if the findings will be similar to the ones observed in the male rats.

\section{Limitation of study}

The study did not look into the possible effect of the ACTs on the offspring of the rats.

\section{Ethical clearance}

Ethical Clearance was obtained from the Ethical committee of the Bingham University Medical School for the study.

\section{Acknowledgements}

None.

\section{Conflict of interest}

The author declares no conflict of interest.

\section{References}

1. WHO. World Malaria Report. Geneva, Switzerland, World Health Organization's: 2016. 186 p.

2. WHO. $Q \& A$ on Artemisinin Resistance. Geneva, Switzerland, World Health Organisation; 2018
3. Mutabingwa TK. Artemisinin-based combination therapies (ACTs): best hope for malaria treatment but inaccessible to the needy. Acta Trop. 2005;95(3):305-315.

4. Hermann M, Untergasser G, Rumpold H, et al. Aging of the male reproductive system. Exp Gerontol. 2000;35(9-10):1267-1279.

5. MacGill R. Testosterone: Function, Dysfunction, and Supplements. Medical News Today. 2016.

6. Boulpaep EL, Boron WF. Medical physiology: a cellular and molecular approach. Philadelphia, Saunders/Elsevier; 2005:1319 p.

7. Nadjm B, Behrens RH. Malaria: An update for physicians. Infect Dis Clin North Am. 2012;26(2):243-259.

8. Pitteloud N, Dwyer AA, DeCruz S, et al. Inhibition of luteinizing hormone secretion by testosterone in men requires aromatization for its pituitary but not its hypothalamic effects: evidence from the tandem study of normal and gonadotropin-releasing hormonedeficient men. J Clin Endocrinol Metab. 2008;93(3):784-791.

9. Fairhurst RM, Nayyar GM, Breman JG, et al. Artemisinin-resistant malaria: research challenges, opportunities, and public health implications. Am J Trop Med Hyg. 2012;87(2):231-241.

10. Beare NA, Lewallen S, Taylor TE, et al. Redefining cerebral malaria by including malaria retinopathy. Future Microbiol. 2011;6(3):349355 .

11. Bartoloni A, Zammarchi L. Clinical aspects of uncomplicated and severe malaria. Mediterr $J$ Hematol Infect Dis. 2012;4(1):e2012026.

12. Kwiatkowski DP. How malaria has affected the human genome and what human genetics can teach us about malaria. Am J Hum Genet. 2005;77(2):171-192.

13. Rijkan MJ, Rijkan JA. Papauorgiou AT, et al. Malaria in Pregnancy; the difficulties in measuring birth weight. BJOG. 2011;118(6):671-678.

14. Raji Y, Osonuga TO, Akinsomisoye OS, et al. Gonadotoxicity evaluation of oral artemisinin derivatives in male rats. $J$ Med Sci. 2005;5(4):303-306.

15. Sehuster CJ, Canfield FD. Influence of quinine on gonadal indices. Endocrinol Metabolism. 1989;8(6):61-75.

16. Adeeko J, Dada MK. Review of side effects and toxicity. Africa Technical; Report Series. 1998:2045.

17. Ganong WF. Review of Medical Physiology. 20th ed. New York: Lange Series, McGraw-Hill Companies; 2001. 870 p.

18. Serge AJ. Follicle-Stimulating Hormone Abnormalities. Endocrinology. 2017

19. Obianime AW, Aprioku JS. Comparative study of artesunate, acts and their combinants on the hormonal parameters of the male guinea-pig. Niger J Physiol Sci. 2009;24(2):101-106. 